\title{
Renormalization-Group Study of Weakly First-Order Phase Transitions
}

\author{
N. Tetradis \\ CERN, Theory Division, \\ CH-1211, Geneva 23, Switzerland
}

\begin{abstract}
We study the universal critical behaviour near weakly first-order phase transitions for a three-dimensional model of two coupled scalar fields - the cubic anisotropy model. Renormalization-group techniques are employed within the formalism of the effective average action. We calculate the universal form of the coarse-grained free energy and deduce the ratio of susceptibilities on either side of the phase transition. We compare our results with those obtained through Monte Carlo simulations and the $\epsilon$-expansion.
\end{abstract}


A quantitative description of weakly first-order phase transitions (which are typically fluctuation driven) is necessary for a wide range of problems. Apart from their relevance to statistical systems (such as superconductors or anisotropic systems, like the one we consider below), weakly first-order phase transitions often appear in the study of high-temperature field theories. As a result they have important implications for cosmology. An example is provided by the electroweak phase transition, which is weakly first order for Higgs boson masses between approximately 40 and $80 \mathrm{GeV}$ [1, 2]. The calculation of the baryon number that can be generated during the electroweak phase transition requires a precise determination of its properties.

A simple example of a system with an arbitrarily weakly first-order phase transition is the cubic anisotropy model [3, 4]. In field-theoretical language, this corresponds to a theory of two real scalar fields $\phi_{a}(a=1,2)$ in three dimensions, invariant under the discrete symmetry $(1 \leftrightarrow-1,2 \leftrightarrow-2,1 \leftrightarrow 2)$. It can also be considered as the effective description of the fourdimensional high-temperature theory with the same symmetry, at energy scales smaller than the temperature [5, 6, 7]. It is relevant for the phase transitions in multi-Higgs extensions of the Standard Model.

Recently, the properties of the weakly first-order phase transitions in this model have been studied in detail, within the $\epsilon$-expansion [8] or through Monte Carlo simulations [9]. In particular, universal amplitudes have been computed, which describe the relative discontinuity of various physical quantities along the phase transition, in the limit when the transition becomes arbitrarily weakly first order. A discrepancy has been observed in the predictions for the universal ratio of susceptibilities $\chi_{+} / \chi_{-}$on either side of the phase transition obtained through Monte Carlo simulations or the $\epsilon$-expansion [10]. More specifically, the Monte Carlo simulations predict $\chi_{+} / \chi_{-}=4.1(5)$, while the first three orders of the $\epsilon$-expansion give $\chi_{+} / \chi_{-}=2.0,2.9,2.3$ respectively.

In this letter we present an alternative approach that employs the exact renormalization group [11]. It is based on the effective average action $\Gamma_{k}$ [12], which is a coarse-grained free energy with an infrared cutoff. More precisely, $\Gamma_{k}$ incorporates the effects of all fluctuations with momenta $q^{2}>k^{2}$, but not those with $q^{2}<k^{2}$. In the limit $k \rightarrow 0$, the effective average action becomes the standard effective action (the generating functional of the 1PI Green functions), while at a high momentum scale (of the order of the ultraviolet cutoff) $k=\Lambda \rightarrow \infty$, it equals the classical or bare action. It is formulated in continuous (Euclidean) space and all symmetries of the model are preserved. The exact non-perturbative flow equation for the scale dependence of $\Gamma_{k}$ takes the simple form of a renormalization-group-improved one-loop equation 13]

$$
k \frac{\partial}{\partial k} \Gamma_{k}[\phi]=\frac{1}{2} \operatorname{Tr}\left[\left(\Gamma_{k}^{(2)}[\phi]+R_{k}\right)^{-1} k \frac{\partial}{\partial k} R_{k}\right] .
$$

The trace involves a momentum integration and summation over internal indices. The relevant infrared properties appear directly in the form of the exact inverse average propagator $\Gamma_{k}^{(2)}$, which is the matrix of second functional derivatives with respect to the fields. There is always only one momentum integration - multiloops are not needed - which is, for a suitable cutoff function $R_{k}\left(q^{2}\right)$ (with $R_{k}(0) \sim k^{2}, R_{k}\left(q^{2} \rightarrow \infty\right) \sim e^{-q^{2} / k^{2}}$ ), both infrared and ultraviolet finite.

The flow equation (1) is a functional differential equation, and an approximate solution requires a truncation. Our truncation is the lowest order in a systematic derivative expansion of $\Gamma_{k}$ [14, 15]

$$
\Gamma_{k}=\int d^{3} x\left\{U_{k}\left(\rho_{1}, \rho_{2}\right)+\frac{1}{2} Z_{k}\left(\partial^{\mu} \phi_{1} \partial_{\mu} \phi^{1}+\partial^{\mu} \phi_{2} \partial_{\mu} \phi^{2}\right)\right\} .
$$

Here $\rho_{a}=\frac{1}{2} \phi_{a} \phi^{a}$ and the potential $U_{k}\left(\rho_{1}, \rho_{2}\right)$ is symmetric under the interchange $1 \leftrightarrow 2$. 
The wave-function renormalization is approximated by one $k$-dependent parameter $Z_{k}$. The truncation of the higher derivative terms in the action is expected to generate an uncertainty of the order of the anomalous dimension $\eta$. (For $\eta=0$ the kinetic term in the $k$-dependent inverse propagator must be exactly proportional to $q^{2}$ both for $q^{2} \rightarrow 0$ and $q^{2} \rightarrow \infty$.) For the model we are considering, $\eta \simeq 0.035$ and the induced error is small. A similar truncation has been employed for the calculation of the equation of state for the $O(N)$-symmetric scalar theory [16]. The result is indeed in agreement with those obtained through alternative methods with an accuracy of order $\eta$.

The evolution equation for the potential results from the substitution of eq. (2) in the exact flow equation (1). The fixed-point structure of the theory is more easily identified if we use the dimensionless renormalized parameters

$$
\tilde{\rho}_{a}=Z_{k} k^{-1} \rho_{a} \quad u_{k}\left(\tilde{\rho}_{1}, \tilde{\rho}_{2}\right)=k^{-3} U_{k}\left(\rho_{1}, \rho_{2}\right) .
$$

The evolution equation for the potential can now be written in the scale-independent form [7]

$$
\frac{\partial}{\partial t} u_{k}\left(\tilde{\rho}_{1}, \tilde{\rho}_{2}\right)=-3 u_{k}+(1+\eta)\left(\tilde{\rho}_{1} u_{1}+\tilde{\rho}_{2} u_{2}\right)-\frac{1}{8 \pi^{2}} L_{0}^{3}\left(\tilde{m}_{1}^{2}\right)-\frac{1}{8 \pi^{2}} L_{0}^{3}\left(\tilde{m}_{2}^{2}\right)
$$

where $t=\ln (k / \Lambda)$. The anomalous dimension $\eta$ is defined as $d \ln Z_{k} / d t=-\eta$ and can be computed starting from the exact flow equation 12, 14. For the model we are considering and the part of the phase diagram we shall study, it is constant $\eta \simeq 0.035$ to a good approximation [7]. The quantities $\tilde{m}_{1,2}^{2}$ are the eigenvalues of the rescaled mass matrix at the point $\left(\tilde{\rho}_{1}, \tilde{\rho}_{2}\right)$

$$
\tilde{m}_{1,2}^{2}=\frac{1}{2}\left\{u_{1}+u_{2}+2 u_{11} \tilde{\rho}_{1}+2 u_{22} \tilde{\rho}_{2} \pm\left[\left(u_{1}-u_{2}+2 u_{11} \tilde{\rho}_{1}-2 u_{22} \tilde{\rho}_{2}\right)^{2}+16 u_{12}^{2} \tilde{\rho}_{1} \tilde{\rho}_{2}\right]^{\frac{1}{2}}\right\},
$$

and we have introduced the notation $u_{1}=\partial u_{k} / \partial \tilde{\rho}_{1}, u_{12}=\partial^{2} u_{k} / \partial \tilde{\rho}_{1} \partial \tilde{\rho}_{2}$, etc. The function $L_{0}^{3}(w)$, as well as the functions $L_{1}^{3}(w)=-d L_{0}^{3}(w) / d w, L_{n+1}^{3}(w)=-1 / n d L_{n}^{3}(w) / d w$ for $n \geq 1$ that we shall encounter in the following, are negative for all values of $w$. Also $\left|L_{n}^{3}(w)\right|$ are monotonically decreasing for increasing $w$ and introduce a threshold behaviour in the evolution. For large values of $\tilde{m}_{a}^{2}$ the last two terms in eq. (勾) vanish and the evolution of $U_{k}$ stops. The above functions have been extensively discussed in refs. [12, 14].

The initial condition for the integration is provided by the bare potential, which is identified with the effective average potential at a very high scale $k=\Lambda$. We use a bare potential of the form

$$
u_{\Lambda}\left(\tilde{\rho}_{1}, \tilde{\rho}_{2}\right)=\frac{1}{2} \lambda_{\Lambda}\left\{\left(\tilde{\rho}_{1}-\kappa_{\Lambda}\right)^{2}+\left(\tilde{\rho}_{2}-\kappa_{\Lambda}\right)^{2}\right\}+\left(1+x_{\Lambda}\right) \lambda_{\Lambda} \tilde{\rho}_{1} \tilde{\rho}_{2}
$$

and $Z_{\Lambda}=1$.

The phase structure of the theory has been discussed in detail in refs. [3, 4, 6, 7]. The phase diagram has three fixed points, which govern the dynamics of second-order phase transitions. They are located on the critical surface separating the phase with symmetry breaking from the symmetric one. The most stable of them corresponds to a system with an increased $O(2)$ symmetry. It can be approached directly from a bare action given by eq. (6) with $x_{\Lambda}=0$. The other two are Wilson-Fisher fixed points, corresponding to two disconnected $Z_{2}$-symmetric theories. One of them can be approached from a bare action with $x_{\Lambda}=-1$, while the second requires $x_{\Lambda}=2$. Flows that start with $-1<x_{\Lambda}<2$ and near the critical surface eventually

\footnotetext{
${ }^{1}$ A redefinition of the fields demonstrates that this choice corresponds to two disconnected $Z_{2}$-symmetric theories [7].
} 
lead to the $O(2)$-symmetric fixed point. For $x_{\Lambda}>2$ or $x_{\Lambda}<-1$ the evolution leads to a region of first order phase transitions. If $x_{\Lambda}$ is chosen slightly larger than 2 or slightly smaller than -1 the phase transitions are weakly first order. As we demonstrate in the following, the evolution first approaches one of the fixed points before a second minimum appears in the potential.

The partial differential equation (4) with $\eta=0.035$ can be integrated numerically through a generalization to the case of two fields of the algorithms presented in ref. [17] for the case of one background field. This approach is straightforward but requires excessive computer power. An alternative solution relies on an approximation that simplifies the form of the potential. We are interested in the case where the minima of the potential are located on the two axes (this requires $\left.x_{\Lambda}>0\right)$. We concentrate on the $\tilde{\rho}_{1}$ axis and consider the region $\tilde{\rho}_{2} \ll 1$. The potential can be expanded as

$$
u_{k}\left(\tilde{\rho}_{1}, \tilde{\rho}_{2}\right)=v_{k}\left(\tilde{\rho}_{1}\right)+f_{k}\left(\tilde{\rho}_{1}\right) \tilde{\rho}_{2}+\ldots
$$

Substituting the above expression in eq. (4) and setting $\tilde{\rho}_{2}=0$ results in an evolution equation for $v_{k}\left(\tilde{\rho}_{1}\right)$. Notice that terms with powers of $\tilde{\rho}_{2}$ higher than the first do not affect the form of this evolution equation.

We approximate the function $f_{k}\left(\tilde{\rho}_{1}\right)$ by a fourth-order polynomial in $\tilde{\rho}_{1}$. This is expected to be a good approximation as the physical behaviour of interest (scale-invariant form of the potential, appearance of a minimum at the origin) is observed for $\tilde{\rho}_{1}<1$ (see fig. 1). Near the origin we have

$$
f_{k}\left(\tilde{\rho}_{1}\right) \simeq m_{s}^{2}+\left(1+x_{s}\right) \lambda_{s} \tilde{\rho}_{1},
$$

with $m_{s}^{2}=v_{1}\left(\tilde{\rho}_{1}=0\right), \lambda_{s}=v_{11}\left(\tilde{\rho}_{1}=0\right)$. The form of the first term in the right-hand side is imposed by the $1 \leftrightarrow 2$ symmetry. The evolution of the $k$-dependent term $x_{s}$ can be calculated from a truncated form of the evolution equation at $\tilde{\rho}_{1}=\tilde{\rho}_{2}=0$ []]

$$
\frac{d x_{s}}{d t}=\frac{1}{8 \pi^{2}}\left(x_{s}+1\right) x_{s}\left(x_{s}-2\right) \lambda_{s} L_{2}^{3}\left(m_{s}^{2}\right) .
$$

Near the minimum of the potential at $\tilde{\rho}_{1}=\kappa \neq 0$ we have

$$
f_{k}\left(\tilde{\rho}_{1}\right) \simeq x_{0} \lambda_{0} \kappa+\left(1+x_{0}\right) \lambda_{0}\left(\tilde{\rho}_{1}-\kappa\right),
$$

with $\lambda_{0}=v_{11}\left(\tilde{\rho}_{1}=\kappa\right)$. Again the form of the first term in the right-hand side is imposed by the $1 \leftrightarrow 2$ symmetry. The evolution of the $k$-dependent term $x_{0}$ can be calculated from a truncated form of the evolution equation at $\tilde{\rho}_{1}=\kappa, \tilde{\rho}_{2}=0$ []]

$$
\frac{d x_{0}}{d t}=\frac{1}{8 \pi^{2}} \frac{6}{\kappa} \frac{x_{0}+\frac{x_{0}^{2}}{4}}{1-\frac{x_{0}}{2}}\left\{L_{1}^{3}\left(2 \lambda_{0} \kappa\right)-L_{1}^{3}\left(x_{0} \lambda_{0} \kappa\right)\right\}+\frac{1}{8 \pi^{2}} x_{0} \lambda_{0}\left\{9 L_{2}^{3}\left(2 \lambda_{0} \kappa\right)+\left(1+x_{0}\right)^{2} L_{2}^{3}\left(x_{0} \lambda_{0} \kappa\right)\right\} .
$$

Matching the two expansions of eqs. (8) and (10) fixes the coefficients of the fourth-order polynomial. We shall check the validity of this approximation in the following.

The numerical integration of the partial differential equation (4) is now feasible, because we have only two independent variables $\left(t, \tilde{\rho}_{1}\right)$ for the function $v_{k}$ (as well as the $k$-dependent parameters $\left.x_{s}, x_{0}\right)$. The expected numerical accuracy of the integration is estimated to be of order $3 \%$ [17].

We are interested in the region $x \geq 2$. The region $x \leq-1$ can be mapped on it through a redefinition of the fields [7]. In fig. 1 we present the evolution of $v_{1}=d v_{k} / d \tilde{\rho}_{1}$, starting at a very high scale $k=\Lambda$ with a bare potential given by eq. (6). All dimensionful quantities are normalized with respect to $\Lambda$. The initial coupling $\lambda_{\Lambda}$ is chosen arbitrarily, while the minimum 
$\kappa_{\Lambda}$ of the bare potential is taken very close to the critical value $\kappa_{c r}$ that separates the phase with symmetry breaking from the symmetric one. For $\left|\delta \kappa_{\Lambda}\right|=\left|\kappa_{\Lambda}-\kappa_{c r}\right| \ll 1$ the system spends a long "time" $t$ of its evolution on the critical surface separating the two phases. The initial value $x_{\Lambda}$ (which determines the initial conditions $x_{s}(\Lambda)=x_{0}(\Lambda)=x_{\Lambda}$ for eqs. (9), (11)) is taken slightly larger than the fixed-point value 2. After the initial evolution (dotted lines) the potential settles down near the Wilson-Fisher fixed point (solid lines). The fixed point of eqs. (9), (11) at $x_{s}=x_{0}=2$ is repulsive f 7 , and $x_{s}, x_{0}$ eventually evolve towards larger values. This forces the potential to move away from its scale-independent form (dashed lines). At some point in the subsequent evolution the curvature of the potential at the origin $v_{1}\left(\tilde{\rho}_{1}=0\right)$ becomes positive. This signals the appearance of a new minimum there, and the presence of a fluctuation-driven first-order phase transition. The evolution of the potential after it moves away from the fixed point is insensitive to the details of the bare potential. It is uniquely determined by $\left|\delta \kappa_{\Lambda}\right|=\left|\kappa_{\Lambda}-\kappa_{c r}\right| \ll 1$ and $\delta x_{\Lambda}=x_{\Lambda}-2 \ll 1$, and, therefore, displays universal behaviour [19.

In the limit $k \rightarrow 0$ and in the convex regions, the rescaled potential $u_{k}$ grows and eventually diverges in such a way that $U_{k}$ becomes asymptotically constant, equal to the effective potential $U=U_{0}$. This is apparent in fig. 2, where the potential along the $\rho_{1}$ axis is plotted. The evolution of the non-convex part of the potential (between the two minima) is related to the issue of the convexity of the effective potential. This part should become flat for $k \rightarrow 0$ [18, 19]. The approach to convexity is apparent in fig. 2, even though we have not followed the evolution all the way to $k=0$. The nucleation rate during the decay of the unstable minimum can be calculated in terms of the potential at an appropriate coarse-graining scale. It is exponentially suppressed by the free energy of the dominant tunnelling configuration. The scale $k$ must be chosen so that the pre-exponential factor, arising from fluctuations around the dominant configuration, is small. An appropriate scale $k_{f}$ for the definition of a universal coarse-grained potential is determined by [19]

$$
\frac{k_{f}^{2}-\left|\left(d^{2} U_{k_{f}} / d \phi_{1}^{2}\right)_{\phi_{1}=\phi_{1 \max }}\right|}{k_{f}^{2}}=0.01
$$

where $\phi_{1 \max }$ corresponds to the top of the barrier. The scale at which we have stopped the evolution in fig. 2 is near $k_{f}$.

The relative magnitude of $\left|\delta \kappa_{\Lambda}\right|=\left|\kappa_{\Lambda}-\kappa_{c r}\right| \ll 1$ and $\delta x_{\Lambda}=x_{\Lambda}-2 \ll 1$ results in different types of evolution. For the type of behaviour depicted in figs. 1 and 2 one must take $\left|\delta \kappa_{\Lambda}\right| \ll \delta x_{\Lambda}$. In the opposite limit, $\left|\delta \kappa_{\Lambda}\right| \gg \delta x_{\Lambda}$, the system leaves the critical surface before $x_{s}, x_{0}$ evolve away from the fixed-point value 2 . As a result, a second minimum never appears at the origin. Instead, the only minimum of the effective potential $U_{0}$ is located either at zero (symmetric phase) or away from it (phase with symmetry breaking), depending on the sign of $\delta \kappa_{\Lambda}$. The resulting phase transition is second order and occurs for $\delta \kappa_{\Lambda}=0$.

We are interested in the universal ratio of susceptibilities $\chi_{+} / \chi_{-}$on either side of the phase transition. This ratio depends on the value of $\left|\delta \kappa_{\Lambda}\right| / \delta x_{\Lambda}$. For $\left|\delta \kappa_{\Lambda}\right| / \delta x_{\Lambda} \gg 1$ we have $x_{s}(k), x_{0}(k) \simeq 2$ during the whole evolution. The universal quantities characterizing the secondorder phase transition are determined by the Wilson-Fisher fixed point. We calculate $\chi_{+} / \chi_{-}$ by integrating the evolution equation and evaluating $d^{2} U_{0} / d \phi_{1}^{2}=\chi^{-1}$ at the minimum, for

\footnotetext{
${ }^{2}$ Despite the presence of $1-x_{0} / 2$ in the denominator in the right-hand side of eq. (9), this equation has a fixed point at $x_{0}=2$.
} 
$\delta \kappa_{\Lambda}=\mp \epsilon$ with $\epsilon \ll 1$. We obtain $\chi_{+} / \chi_{-}=4.1$. This value should be compared with the value $\chi_{+} / \chi_{-}=4.3$, calculated with the same method for the one-field, $Z_{2}$-symmetric theory (the Ising model) without any polynomical approximations for the form of the potential [16]. The difference is due to the approximation of the function $f_{k}\left(\tilde{\rho}_{1}\right)$ in eq. (可) by a fourth-order polynomial. An error of this magnitude is typically induced by the truncation of the potential to a polynomial form [14, 16]. We have verified this conclusion by approximating the function $f_{k}\left(\tilde{\rho}_{1}\right)$ by eq. (8) without using eq. (10). This cruder approximation results in $\chi_{+} / \chi_{-}=4.0$.

The evaluation of the same quantity through the $\epsilon$-expansion or an expansion at fixed dimension gives $\chi_{+} / \chi_{-}=4.8(3)$ [20], whereas experimental information gives $\chi_{+} / \chi_{-}=4.3(3)$ [20]. The difference with the results of our method is due to the omission of higher derivative terms in the effective average action of eq. (2). This confirms our expectation, discussed earlier, that the error induced by this approximation is determined by the anomalous dimension, which is small for our model $(\eta \simeq 0.035)$.

For $\left|\delta \kappa_{\Lambda}\right| / \delta x_{\Lambda} \ll 1$ the potential develops a second minimum at the origin during the later stages of the evolution. The phase transition is approached by fine tuning $\delta \kappa_{\Lambda}$, so that the two minima have equal depth for $k=0$. In fig. 3 we plot the difference in energy density $\Delta U$ between the minimum away from the origin and the one at the origin, as a function of the scale $k$, for four values of $\delta \kappa_{\Lambda}$. The numerical integration of the evolution equation is difficult for $k \rightarrow 0$ because of the singularity structure of $L_{n}^{3}(w)$ [18, 19]. As a result the curves of fig. 3 must be extrapolated to $k=0$. Line (a) corresponds to a system in the symmetric phase, line (d) to one in the phase with symmetry breaking. Lines (b) and (c) correspond to a system very close to the phase transition. The evolution of $\chi_{+} / \chi_{-}$for the same parameters is depicted in fig. 4 .

The extrapolated value of $\chi_{+} / \chi_{-}$for $k=0$ near the phase transition is expected to lie in the interval $(1.5,2)$. We have used numerical fits of various curves for $\Delta U$ and $\chi_{+} / \chi_{-}$in the vicinity of the phase transition in order to perform the extrapolation to $k=0$. The expected value for $\chi_{+} / \chi_{-}$is 1.7 .

The main sources of error in our calculation are the derivative expansion of eq. (2) and the approximation of $f_{k}\left(\tilde{\rho}_{1}\right)$ in eq. (7) by a fourth-order polynomial in $\tilde{\rho}_{1}$. We argued earlier that the error induced by the derivative expansion is related to the small anomalous dimension $\eta \simeq 0.035$. We also checked above the error induced by the polynomial appoximation of the potential. For the second-order phase transition, two polynomial approximations give $\chi_{+} / \chi_{-}=4.0$ and 4.1 , while no polynomial approximation gives $\chi_{+} / \chi_{-}=4.3$ in the order of the derivative expansion we are working [16]. The numerical integration and the extrapolation used in the calculation of $\chi_{+} / \chi_{-}$induce smaller errors. The $\epsilon$-expansion or an expansion at fixed dimension gives $\chi_{+} / \chi_{-}=4.8(3)$ [20], whereas experimental information gives $\chi_{+} / \chi_{-}=4.3(3)$ [20]. In order to be conservative, we use as a maximum total error the difference between our result $\chi_{+} / \chi_{-}=4.1$ for the case of the second-order phase transition and the central value $\chi_{+} / \chi_{-}=4.8$ of the result from the $\epsilon$-expansion.

Our result

$$
\chi_{+} / \chi_{-}=1.7(7)
$$

is depicted by a horizontal band in fig. 4. It is in good agreement with the predictions of the $\epsilon$-expansion $\left(\chi_{+} / \chi_{-}=2.0,2.9,2.3\right.$ for the first three orders $)$ [8, 10]. It disagrees, however, with the lattice result presented in refs. [9, 10] $\left(\chi_{+} / \chi_{-}=4.1(5)\right)$. The values favoured by the lattice calculation correspond to a theory well into the phase with symmetry breaking in our calculation (line (d) in figs. 3 and 4). However, it should be noted that, according to the authors of ref. [10], the error of the lattice result "should be taken with a grain of salt". 
In summary, we presented a method that can provide a quantitative description of the universal behaviour near weakly first-order phase transitions. It is based on the calculation of a coarse-grained free energy through an exact flow equation. Fixed points in the evolution, the appearance of new minima in the potential, and the universal properties of the resulting fluctuation-driven first-order phase transitions can be studied in detail. We calculated the universal ratio of susceptibilities $\chi_{+} / \chi_{-}$on either side of the first-order phase transition in a two-scalar model . 


\section{References}

[1] K. Kajantie, M. Laine, K. Rummukainen and M. Shaposhnikov, Phys. Rev. Lett. 77, 2887 (1996); V.A. Rubakov and M.E. Shaposhnikov, preprint CERN-TH/96-13, hep-ph/9603208; K. Jansen, preprint DESY-95-169, hep-lat/9509018.

[2] N. Tetradis, Nucl. Phys. B 488, 92 (1997).

[3] J. Rudnick, Phys. Rev. B 18, 1406 (1978).

[4] A. Aharony, in: Phase Transitions and Critical Phenomena, vol. 6, eds. C. Domb and M.S. Green, Academic Press (1976); D.J. Amit, Field Theory, the Renormalization Group, and Critical Phenomena, World Scientific (1984).

[5] N. Tetradis and C. Wetterich, Nucl. Phys. B 398, 659 (1993).

[6] M. Alford and J. March-Russell, Nucl. Phys. B 417, 527 (1994).

[7] S. Bornholdt, N. Tetradis and C. Wetterich, Phys. Lett. B 348, 89 (1995); Phys. Rev. D 53, 4552 (1996); S. Bornholdt, P. Büttner, N. Tetradis and C. Wetterich, preprint CERN$\mathrm{TH} / 96-67$, cond-mat/9603129.

[8] P. Arnold and L.G. Yaffe, Phys. Rev. D 55, 7760 (1997); P. Arnold and Y. Zhang, Phys. Rev. D 55, 7776 (1997).

[9] P. Arnold and Y. Zhang, Nucl. Phys. B 501, 803 (1997).

[10] P. Arnold, S.R. Sharpe, L.G. Yaffe and Y. Zhang, Phys. Rev. Lett. 78, 2062 (1997).

[11] K.G. Wilson, Phys. Rev. B 4, 3174 and 3184 (1971); K.G. Wilson and I.G. Kogut, Phys. Rep. 12, 75 (1974); F.J. Wegner, in: Phase Transitions and Critical Phenomena, vol. 6, eds. C. Domb and M.S. Green, Academic Press (1976).

[12] C. Wetterich, Nucl. Phys. B 352, 529 (1991); Z. Phys. C 57, 451 (1993); ibid. C 60, 461 (1993); Phys. Lett. B 301, 90 (1993).

[13] C. Wetterich, Phys. Lett. B 301, 90 (1993).

[14] N. Tetradis and C. Wetterich, Nucl Phys. B 422, 541 (1994).

[15] T. Morris, Nucl. Phys. B 495, 477 (1997).

[16] J. Berges, N. Tetradis and C. Wetterich, Phys. Rev. Lett. 77, 873 (1996).

[17] J. Adams, J. Berges, S. Bornholdt, F. Freire, N. Tetradis and C. Wetterich, Mod. Phys. Lett. A 10, 2367 (1995).

[18] A. Ringwald and C. Wetterich, Nucl. Phys. B 334, 506 (1990); N. Tetradis and C. Wetterich, Nucl. Phys. B 383, 197 (1992).

[19] J. Berges and C. Wetterich, Nucl. Phys. B 487, 675 (1997); J. Berges, N. Tetradis and C. Wetterich, Phys. Lett. B 393, 387 (1997).

[20] J. Zinn-Justin, Quantum Field Theory and Critical Phenomena, Oxford Science Publications (1989). 


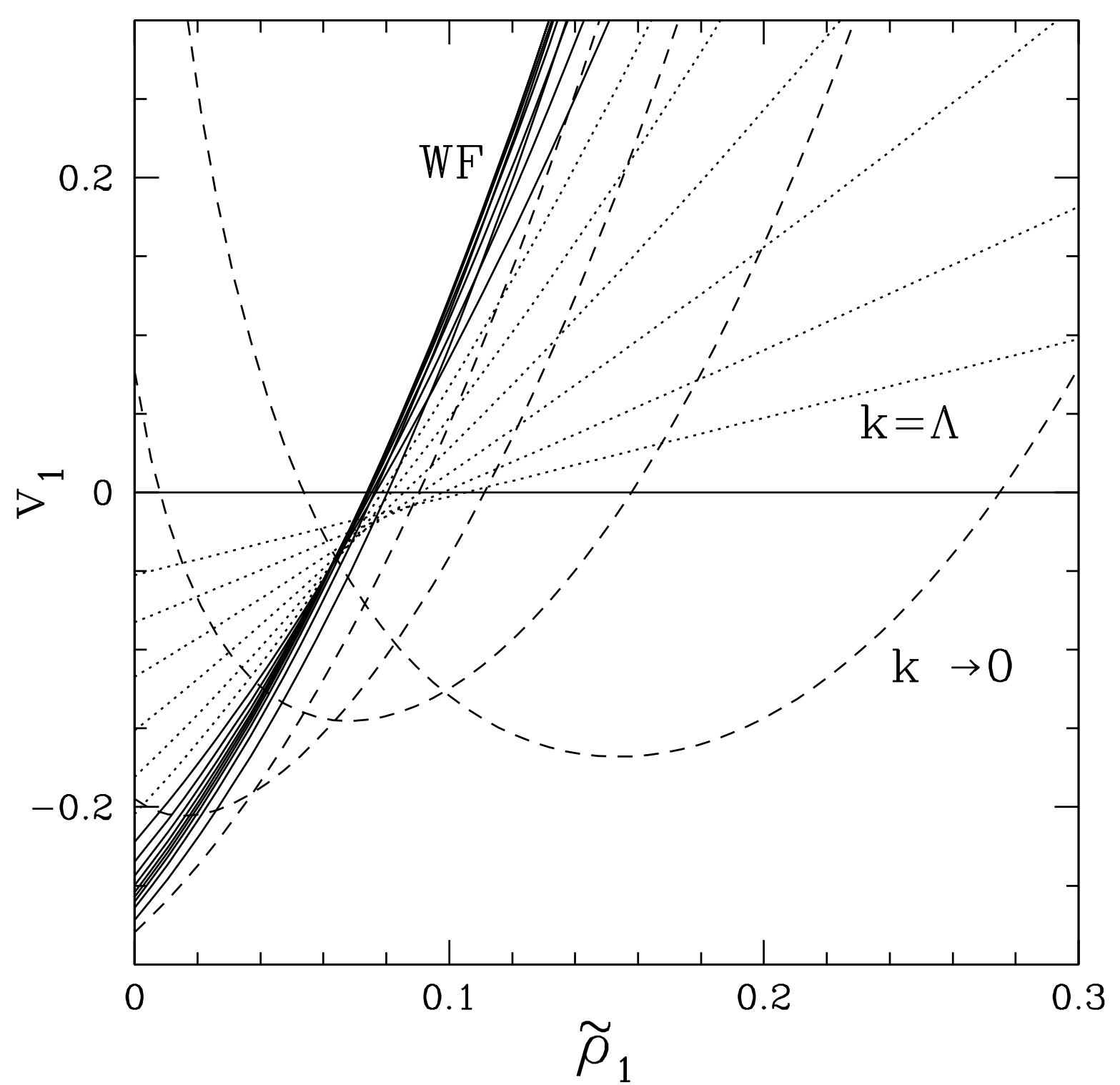

Fig. 1: The derivative of the rescaled potential along the $\rho_{1}$ axis, as the coarse-graining scale is lowered. 


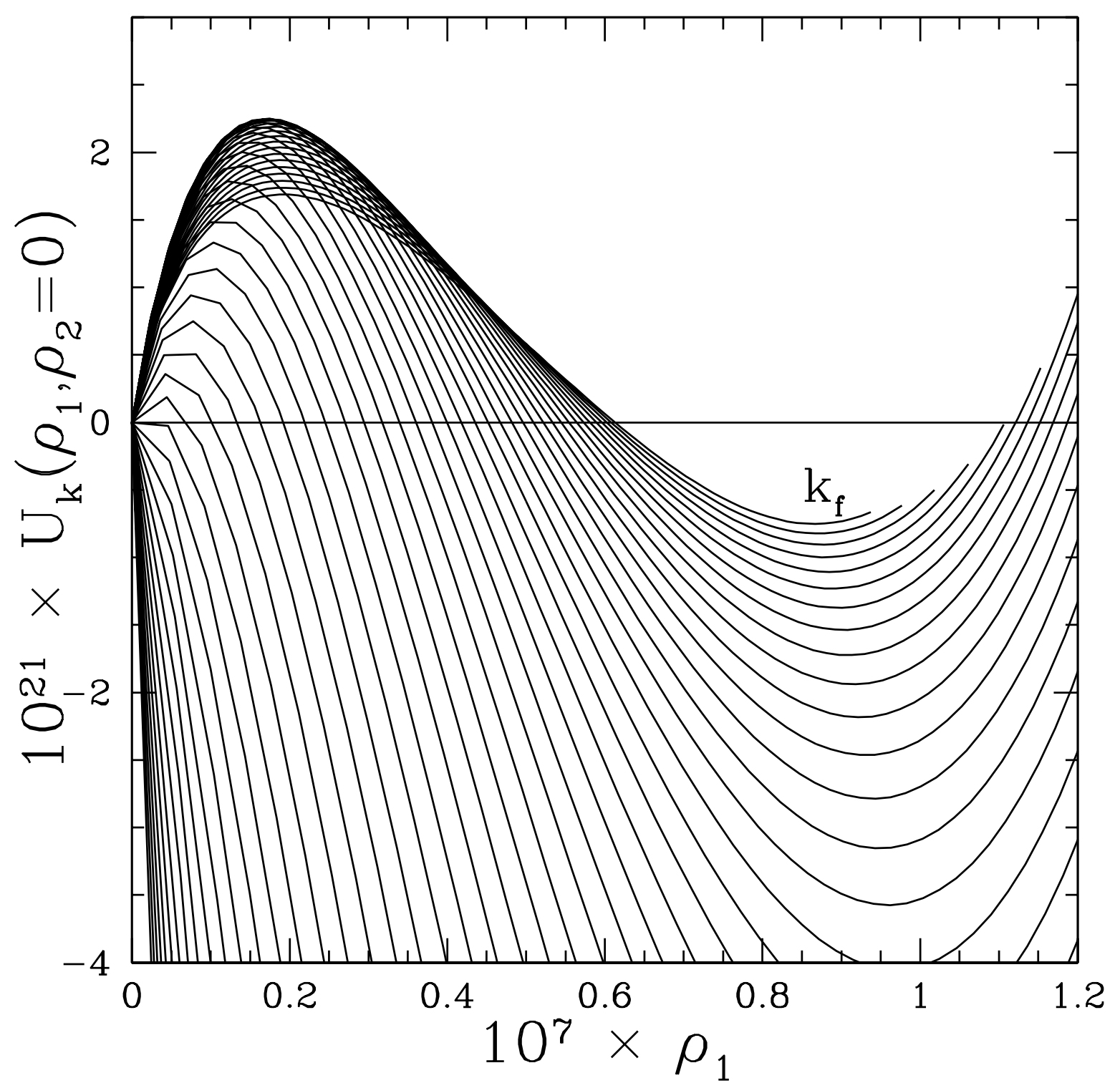

Fig. 2: The potential along the $\rho_{1}$ axis, as the coarse-graining scale is lowered. 


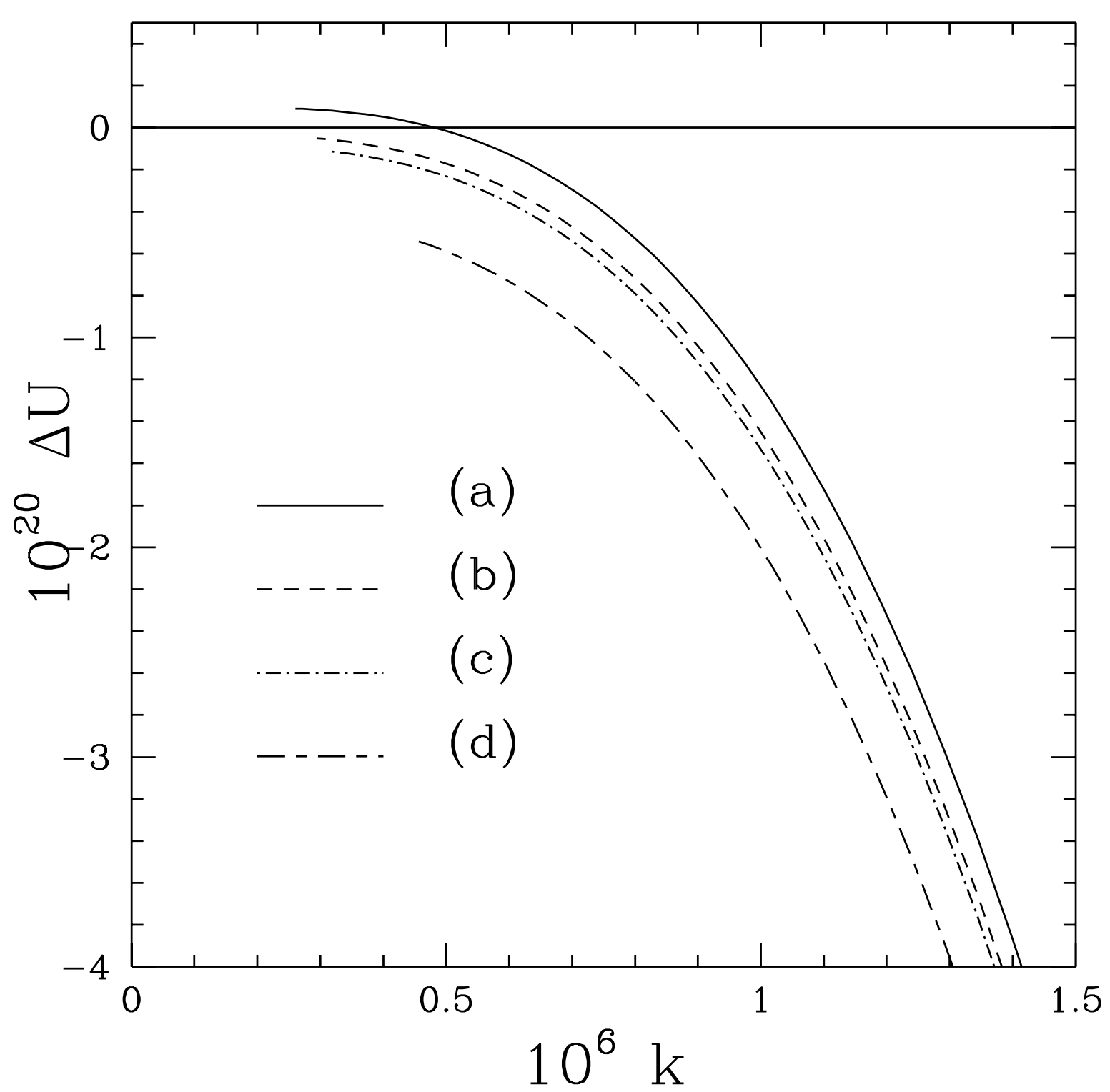

Fig. 3: The difference in energy density between the two minima of the potential, as a function of the coarse-graining scale, near the phase transition. Line (a) corresponds to a system in the symmetric phase, line (d) to one in the phase with symmetry breaking. Lines (b) and (c) correspond to a system very close to the phase transition. 


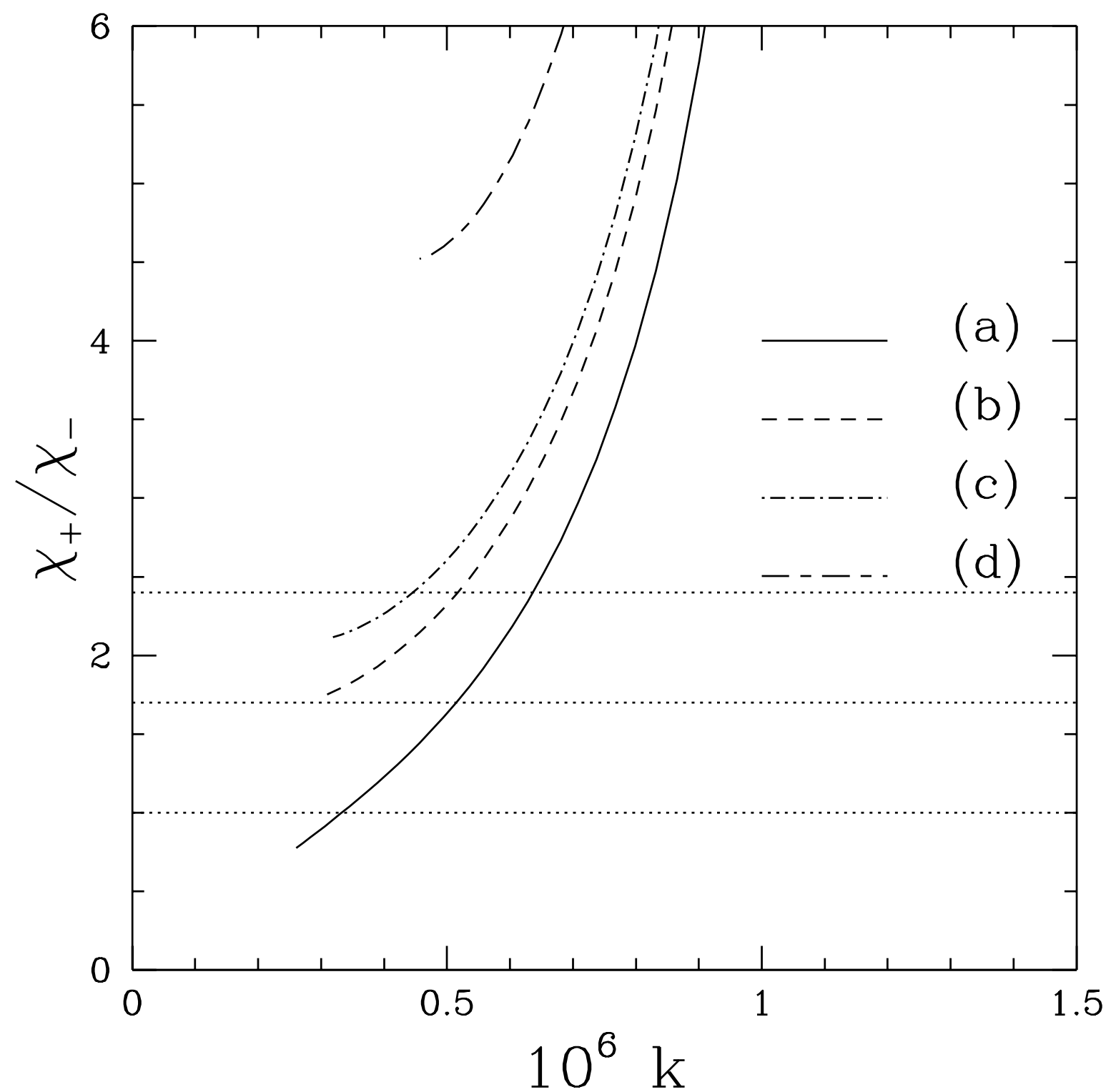

Fig. 4: Analogous to fig. 3 for the ratio of susceptibilities $\chi_{+} / \chi_{-}$. 